\title{
Slamming impact loads on high-speed craft sections using two-dimensional modelling
}

\author{
J. Camilleri, D.J. Taunton \& P. Temarel \\ Fluid Structure Interactions Group, University of Southampton, Southampton, UK
}

\begin{abstract}
The slamming impact loads experienced by high-speed craft travelling in rough seas are numerically investigated by analysing the idealized problem of a two-dimensional rigid wedge impacting an initially calm water surface. We are using the commercial CFD software Star CCM+, which is based on the Finite Volume (FV) method and an interface capturing scheme of the Volume-of-Fluid (VOF) type. The set-up of the numerical model is described in detail. The influence of mesh size, time step, and other numerical parameters such as inner iterations and relaxation factors, as well as three-dimensional modelling, on the solution is studied in a systematic manner. Comparisons against published experimental data show favorable agreement.
\end{abstract}

\section{INTRODUCTION}

Slamming in rough seas is of concern for a wide range of ship and offshore structures, particularly for small high-speed craft, such as patrol, military and rescue craft, which often have to travel at the highest speed possible. The craft frequently launches off waves, emerging from the water, and then violently impacts onto the free surface with high relative velocity. This may lead to crew injuries and structural failure, either due to fatigue loading or catastrophic failure during an extreme event.

Slamming induced loads constitute a significant portion of the design loads and, thus, need to be carefully considered for safe, reliable, and efficient structural design. An accurate prediction method for the loads and responses is, therefore, crucial. Traditionally, hull-water impacts have been investigated by analysing the idealized problem of a twodimensional hull section such as a bow or V-shaped section impacting an initially calm water surface.

Significant amount of research has been reported in the literature on the two-dimensional (2D) water entry problem. Generally, the structure has been assumed rigid and the hydrodynamic impact loads and structural response obtained without coupling, neglecting the effect of the flexibility of the structure on the fluid loading. Pioneering research was carried out by Wagner (1932) who applied potential flow theory to estimate the pressure distribution on rigid wedges for constant velocity impact. Since Wagner (1932), the problem has been approached using a wide range of analytical and numerical methods.
Zhao et al. (1996) applied a nonlinear Boundary Element Method that accounts for flow separation to study the water entry of a wedge and a bow section. This $2 \mathrm{D}$ model was found to overpredict the pressures for the wedge section. The authors were able to quantify the three-dimensional (3D) effects and successfully correct their results. Muzaferija et al. (1998) used a FV method with a free-surface capturing model of the VOF type. This method is well capable of predicting the large free surface deformations, including overturning and breaking waves. However, a fine grid is needed to capture all the details of the jet. The numerical model was validated against the experimental results of Zhao et al. (1996). The size of the numerical domain (tank) and three-dimensional effects were found to have a significant influence on the results. Sames et al. (1999) adopted a similar approach to predict the impact loads on the bow section of a containership. The motion history of water entry was found to have a significant influence on the pressures. The authors conclude that coupling with a rigid body motion solver is required to achieve realistic design pressures. Similar conclusions were reached by Reddy et al. (2002). Recently developed modelling techniques applied to the water entry problem include Smoothed Particle Hydrodynamics (SPH) methods, e.g. see Oger et al. (2006), Veen \& Gourlay (2012) and explicit Finite Element methods, e.g. see Stenius et al. (2006), Wang \& Guedes Soares (2012). Brizzolara et al. (2008) applied a wide range of potential and viscous CFD methods to assess their suitability for simulating a bow section impacting at various speeds and heel angles. 
The 2D slamming problem has also been investigated experimentally. Aarsnes (1996) carried out drop tests using a wedge and a bow section from different heights and at different roll angles to investigate the pressure distribution and resulting impact force. Yettou et al. (2006) studied the influence of deadrise angle, wedge mass and drop height on the pressure distribution and dynamic behavior of a free-falling wedge. All three parameters were found to have a significant influence on the wedge velocity, and thus, the impact loads. Tveitnes et al. (2008) measured the vertical hydrodynamic force on rigid wedge-shaped sections during constant velocity entries. Lewis et al. (2010) presented a comprehensive set of high-quality experimental data for a free-falling $25^{\circ}$ deadrise wedge for two drop heights and wedge mass values. The results are in line with those of Yettou et al. (2006) namely, pressure increases with increasing drop height and wedge mass. A detailed experimental uncertainty analysis is presented making the data highly suitable for validation of numerical models.

The present paper investigates modelling of a $2 \mathrm{D}$ rigid wedge impacting an initially calm water surface using the commercial CFD software Star $\mathrm{CCM}+$. The influence of mesh size, time step, and other numerical parameters, as well as the influence of $3 \mathrm{D}$ effects, on the solution is studied in a systematic manner. The numerical results are compared against the experimental drop test data of Lewis et al. (2010), showing good agreement for different drop heights and wedge mass.

\section{NUMERICAL SOLUTION METHOD}

The computations presented in this paper are performed using the commercial CFD software Star $\mathrm{CCM}+$. It uses the FV method to transform the continuous governing equations into a system of algebraic equations that can be solved numerically. The free surface is modeled using an interface capturing method of the Volume-of-Fluid (VOF) type. Motion of the rigid body i.e., the wedge, is determined as part of the solution by solving the rigid body equations of motion and the mathematical model of fluid flow in a coupled manner.

The starting point for the computations of incompressible viscous fluid flows is the NavierStokes (NS) equations i.e., the mass and momentum conservation equations. In the present approach, a single grid that extends over the entire computational domain and moves with the rigid body (moving-grid approach) is adopted. Thus, the space conservation law, describing the conservation of volume when the Control Volumes (CVs) change their shape or position with time, must also be satisfied.
Air and water are considered as two immiscible components of a single fluid, separated by an interface called the free surface. The location of the free surface (spatial distribution of air and water) is defined in terms of a scalar variable called the volume fraction, found by solving an additional transport equation. The fluid properties are calculated using the physical properties of the two phases and their volume fractions. In Star CCM+, the VOF multiphase model is used to model the free surface and its deformations.

To complete the mathematical model, initial and Boundary Conditions (BC) are specified. Initial conditions describe the state of the flow at start time, whereas boundary conditions describe the state of the flow on the domain boundaries. The latter can be either Dirichlet or Neumann type. Dirichlet conditions specify the actual value of the flow variable (inlet and wall BCs) whereas Neumann conditions specify its gradient (outlet and symmetry BCs).

The above equations close the mathematical model of flow with free surfaces computed using moving grids. The motion of rigid bodies is assumed to be governed by Newton's laws of motion. The external forces acting on the body consist of flow-induced forces (pressure and shear contributions) obtained by solving the mathematical model of fluid flow, and other body forces such as the gravitational force. In Star CCM+, the Dynamic Fluid Body Interaction (DFBI) model is used to simulate rigid body motion.

The spatial solution domain is first divided into a finite number of contiguous CVs, collectively known as the 'mesh' or 'grid'. Each CV surrounds a point at which the dependent variable is evaluated. The CVs can be of any polyhedral shape, allowing for local refinements in the regions of interest. The time interval of interest is also subdivided into a finite number of small time steps. Volume and surface integrals in the governing equations are approximated for each $\mathrm{CV}$ using the mid-point rule. Linear interpolation and linear shape functions are generally used to compute cell face values and gradients from known information at the CV centers. All of these approximations are of second order accuracy. An implicit time-marching scheme is used as it allows larger time steps to be used and provides better stability compared to explicit schemes. Implicit schemes, however, require solutions of system of coupled non-linear equations, which are computationally expensive. The time derivative is approximated using either first-order Euler or second-order three-time-levels scheme, with the latter being more accurate but for flows with free surfaces it has a constraint on the time step size, as discussed in section 3.2.1. 
The discretization of the convective part of the volume fraction equation should neither produce numerical diffusion nor unbounded values of volume fraction. In Star CCM+, the HRIC convection discretization scheme of Muzaferija et al. (1998) is used to achieve a sharp interface and avoid unphysical solutions.

The numerical solution of the system of coupled non-linear equations is obtained in a segregated iterative manner following the SIMPLE algorithm. The linearized momentum equations are solved first using prevailing pressure and mass fluxes to yield an approximate velocity field. The pressurecorrection equation, derived from the discretized continuity equation, is then solved to correct the pressure and velocity fields such that continuity is satisfied. Additional conservation equations, such as for volume fraction are then solved. The process is repeated until all the nonlinear and coupled equations are satisfied within an acceptable tolerance (inner iterations) after which the solution advances to the next time step. In the SIMPLE algorithm, relaxation techniques, governing the extent to which the old solution is supplanted by the newly computed solution are employed. The choice of number of inner iterations and underrelaxation factor values (for pressure, velocity, and other scalar variables) can have a significant influence on the convergence and stability of the solution. The greater the under-relaxation factor used the more of the new solution is used in the calculation. Low under-relaxation factors help maintains numerical stability in the solution at the expense of slower solution convergence rates and vice-versa. Optimal under-relaxation factor values are problem dependent and systematic studies should be performed to determine these values. The number of iterations should be such that the solution is converged within each time step. Small physical time steps imply that the solution is not changing much from one time step to the other, thus, requiring less inner iterations per time step. However, for strongly coupled problems, such as free surface flows with body motion, more inner iterations (around 10) are generally needed to achieve convergence. This can be judged by monitoring the magnitude of the residuals drop within each time step.

For coupled simulations of fluid flow and flowinduced body motion the iteration loop is extended to update the body position. The updated flow fields are used to estimate the flow-induced forces on the body. The governing equations of rigid motion are then solved to find the new body position and adapt the grid accordingly. Since the boundary conditions also change position with time, they need to be adapted at each time step. For instance, the hydrostatic pressure on the bottom boundary increases as the wedge is falling.

\section{WATER ENTRY OF A RIGID WEDGE}

\subsection{Experimental drop tests of Lewis et al. (2010)}

Lewis et al. (2010) carried out a detailed experimental investigation into the impact of a free falling $25^{\circ}$ deadrise angle rigid wedge with water. A schematic diagram of the experimental set-up is shown in Figure 1, and the tank and wedge dimensions are given in Table 1. The wedge was dropped from two different heights, $0.5 \mathrm{~m}$ and $0.75 \mathrm{~m}$ and for each drop height two wedge masses were tested, $23.3 \mathrm{~kg}$ and $33.3 \mathrm{~kg}$. The wedge impact velocities, calculated using the high speed camera images are $2.78 \mathrm{~m} / \mathrm{s}$ and $3.58 \mathrm{~m} / \mathrm{s}$ for 0.5 and $0.75 \mathrm{~m}$ drop height, respectively.

The time-varying pressure distribution on the wedge bottom during impact is measured using six pressure transducers evenly distributed as shown in Figure 2. Wedge acceleration and position are also measured. The experimental pressure and acceleration time histories for a $0.5 \mathrm{~m}$ drop height and a $23.3 \mathrm{~kg}$ wedge mass are shown in Figure 3 . It is important to note that the pressure and acceleration data is low pass filtered using a cut-off frequency of $1000 \mathrm{~Hz}$ and $250 \mathrm{~Hz}$ respectively to remove any high frequency noise in the signals. A high-speed camera is also used to capture the impact and subsequent formation of the jet.

\subsection{Model generation}

In this paper, only one test configuration is analysed in detail, a $23.3 \mathrm{~kg}$ wedge dropped form a

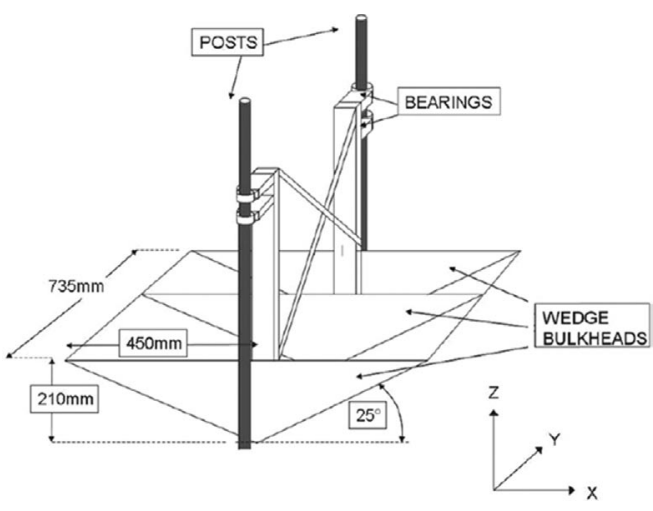

Figure 1. Experimental set-up (Lewis et al. 2010).

Table 1. Tank and wedge dimensions; L-length; W-width; D-depth.

\begin{tabular}{ll}
\hline Tank $\mathrm{L} \times \mathrm{W} \times \mathrm{D}(\mathrm{m})$ & Wedge $\mathrm{L} \times \mathrm{W} \times \mathrm{D}(\mathrm{m})$ \\
\hline $5.8 \times 0.75 \times 0.59$ & $0.944 \times 0.735 \times 0.22$ \\
\hline
\end{tabular}




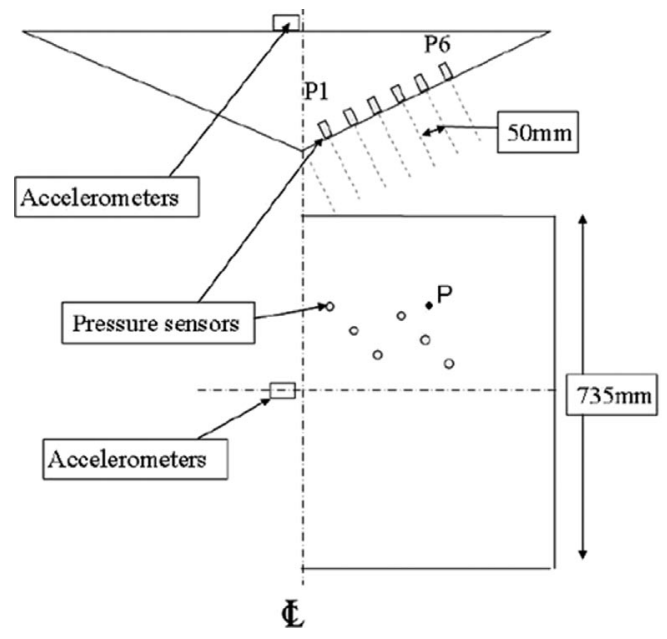

Figure 2. Position of sensors on the wedge (Lewis et al. 2010).

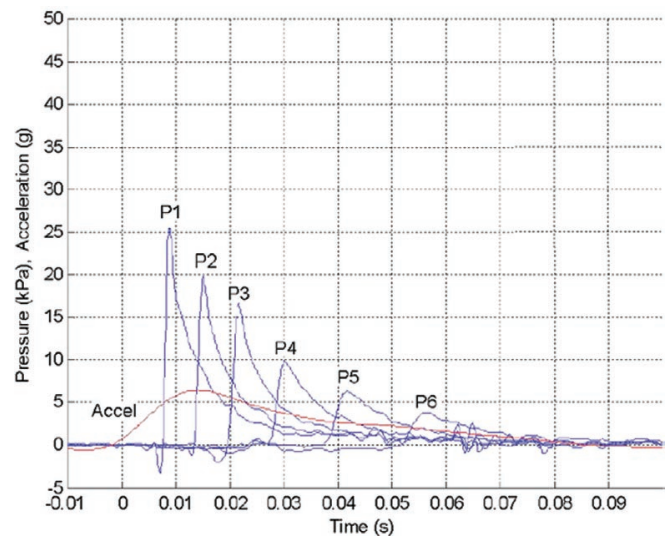

Figure 3. Pressure and acceleration time history for a $0.5 \mathrm{~m}$ drop height with a wedge mass of $23.3 \mathrm{~kg}$ (Lewis et al. 2010).

height of $0.5 \mathrm{~m}$. However, the peak pressures for the other configurations are also presented.

The $2 \mathrm{D}$ discretization of the wedge and tank is shown in Figure 4. Only half of the wedge is considered, and a symmetry boundary condition is imposed on the geometrical symmetry plane of the wedge. The size of the numerical tank is extended to limit the influence of the domain boundaries on the solution. The half-width of the tank is $4.0 \mathrm{~m}$ (y-direction), the height is $5.0 \mathrm{~m}$ (z-direction) and the initial water depth is $2.36 \mathrm{~m}$. The geometrical model has an in-plane thickness (x-direction) of $10 \mathrm{~mm}$ with a symmetry condition applied at the front and back faces to ensure 2D flow. A noslip wall condition is applied on the wedge walls

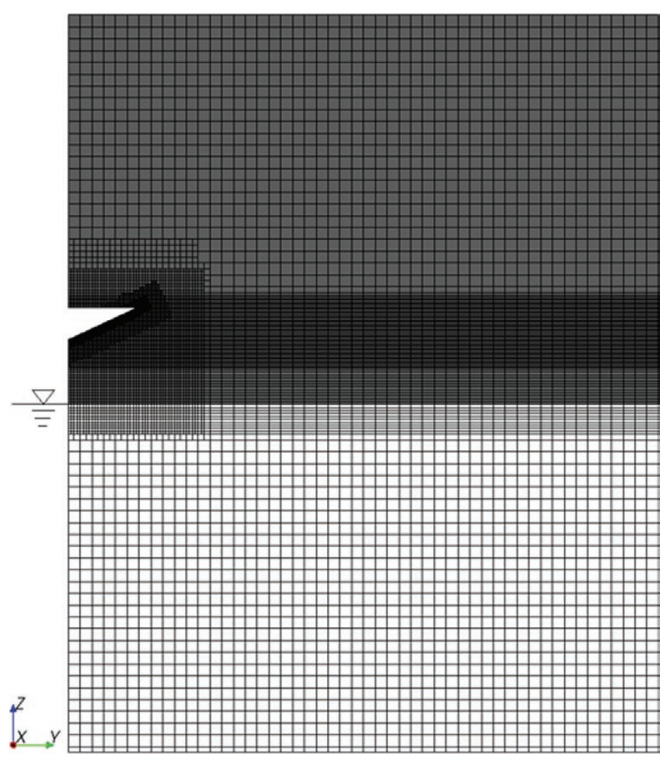

Figure 4. 2D model of wedge and tank, before freefall; air depicted by the dark shaded region; medium grid (Table 4).

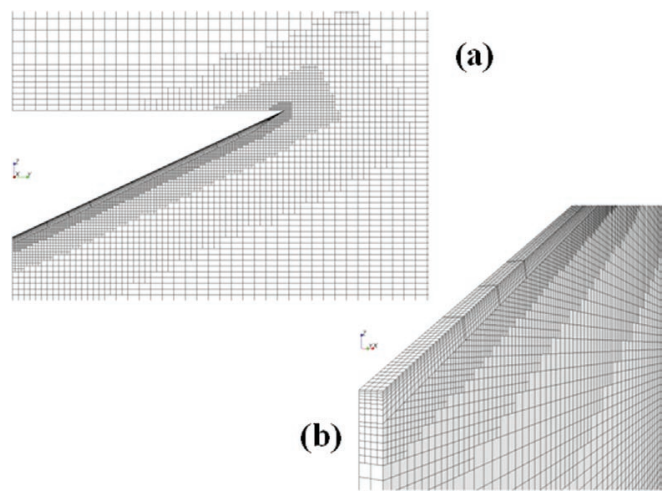

Figure 5. Views of the mesh illustrating (a) local refinements and (b) prism layers; medium grid (Table 4).

and the (right-hand-side) tank wall. The bottom boundary is set to velocity inlet and the top boundary to pressure outlet with prescribed velocity and pressure profiles.

The single mesh extending over the whole computational domain is trimmed Cartesian, with local refinements in the vicinity of the wedge bottom and on the free surface to accurately resolve the highly localized peaked pressure distribution and the free surface profile, respectively shown in Figure 4 and Figure 5. Orthogonal prismatic cells (prism layers) are used near the wedge bottom surface to accurately resolve the near wall flow features such as jet 
formation and flow separation, which can have a significant influence on the pressure and velocity fields (see Figure 5b). The refined mesh extends well below the wedge apex to accurately capture the free surface, during time interval of interest. The mesh gets coarser towards the tank walls as shown in Figure 4. This is achieved by setting up volumetric controls with varying levels of refinement.

Preliminary simulations with a $0.5 \mathrm{~m}$ drop height were found to overestimate the impact velocity. This is most likely to be due to mechanical friction between the bearings and cylindrical posts in the experiments (see Figure 1), which is not accounted for in the simulations. The initial position of the wedge keel was thus set to $0.44 \mathrm{~m}$ above the water surface to match the experimental impact velocity.

The flow is assumed to be viscous and incompressible. Furthermore, a laminar flow model is employed, meaning that the Navier-Stokes equations are solved rather than the ReynoldsAveraged-Navier-Stokes (RANS) equations. Turbulence effects are believed to be small compared to the large slamming impact pressures which occur during the initial stages of water entry (Piro $\&$ Maki 2013). The free surface is modelled using the VOF multiphase and VOF wave models. The VOF wave model is used to simulate surface gravity waves on a light fluid-heavy fluid interface. Here a flat wave with zero fluid velocity is used to represent the calm water surface. The VOF wave model provides the necessary information to initialize the volume fraction, pressure and velocity fields (initial conditions), and to describe the flow at the domain boundaries (boundary conditions). The DFBI model is used to simulate the motion of the rigid body and grid in response to the flow induced forces and gravity. In the computations, motion is restricted to the negative z-direction only (vertical). All computations were carried out with an implicit unsteady time-stepping scheme and a constant time step size. The choice of time step, grid size and other numerical parameters such as inner iterations and under relaxations factors has been performed in a systematic manner as discussed in the following.

\subsubsection{Effect of time step size}

The choice of time step size is mainly governed by the time scales of the physical phenomena of interest i.e., the highly localized (in time) peaked pressure distributions. Furthermore, the time step size must also be chosen in relation to the grid size, to avoid instabilities in the solution. The Courant number is a helpful indication for selecting the time step size. It describes how far the fluid travels during one time step relative to the mesh size. For flows with free surfaces, and a second-order time integration scheme, the Courant number has to be less than 0.5.
If the free surface moves by more than half a cell per time step, the HRIC scheme can lead to local disturbance in the flow and even divergence.

A systematic study has been performed to find an optimum time step size for the original mesh developed i.e., the medium grid shown in Figure 4. Three different time steps are studied. Their influence on the maximum Courant number (CFL), CPU time, pressure and vertical force are presented in Table 2, Figure 6 and Figure 7 respectively. It is important to

Table 2. Effect of time step on Courant number and CPU time; medium grid.

\begin{tabular}{llc}
\hline Time step size $(\mathrm{s})$ & Courant number & CPU time* $(\mathrm{h})$ \\
\hline 0.0001 & $\approx 2.0$ & 0.8 \\
0.00005 & $\approx 1.0$ & 4.7 \\
0.00002 & $\approx 0.5$ & 11.4 \\
\hline
\end{tabular}

* Simulations were run using 48 cores of the IRIDIS 4 supercomputer at the University of Southampton running at $2.6 \mathrm{GHz}$.

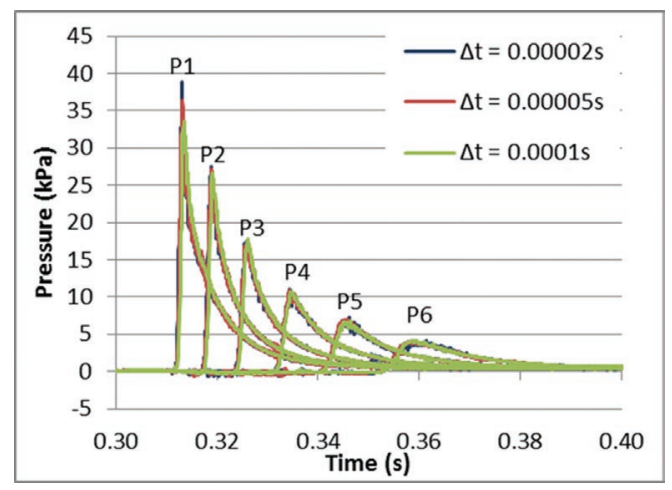

Figure 6. Effect of time step size on the pressure time history for all the pressure sensors; medium grid (Table 4).

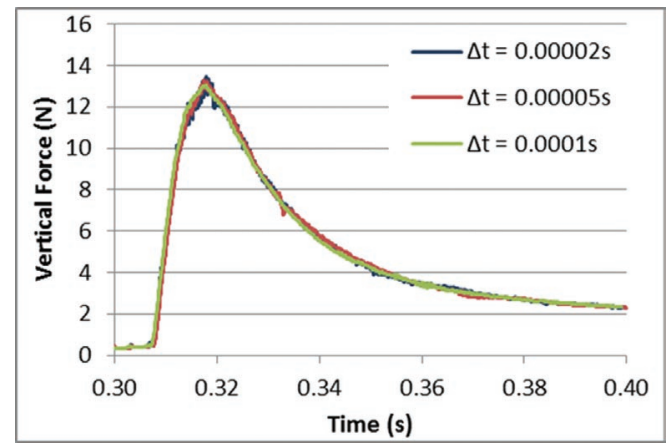

Figure 7. Effect of time step size on the time history of vertical force on the wedge; medium grid (Table 4). 
note that the computed pressures are surface averaged over a circular area $9 \mathrm{~mm}$ in diameter; equivalent to the diameter of the pressure sensors used in the experiments. Furthermore, the presented vertical force time histories are filtered using a 10-point moving average to remove high frequency noise, particularly during the initial stages of water entry, to enable better comparison and analysis.

The pressure time histories show good agreement indicating that the chosen time steps are sufficiently fine to resolve the highly localized impact pressures, except for the peak pressure at P1. Sensor P1 is located very close to the impact region (see Figure 2) where the fluid is suddenly accelerated to very high velocities. That is to say, in this location the pressure is high and rising very sharply, which is difficult to capture in the experiments, as well as the simulations. The Courant condition might also be violated in this region leading to local flow instability. The force plots also show good agreement both in terms of shape and magnitude. Further simulations are thus carried out using a $0.00005 \mathrm{~s}$ time step size even though the Courant condition is violated. However, this occurs in a very small number of cells (around 10) and the solution is stable. Even though a $0.0001 \mathrm{~s}$ time step size provides reasonably good predictions, it is important to ensure that the Courant condition is not violated $(\mathrm{CFL} \approx 2.0$ ) to avoid instabilities. Furthermore a $0.00002 \mathrm{~s}$ time step size is too fine and requires significant computational power.

\subsubsection{Effect of numerical parameters}

As discussed in section 2, the numerical stability and convergence of the solution depends on a number of parameters, such as temporal discretization, relaxation factors and inner iterations. The influence of these parameters on the solution is studied in a systematic manner, by changing their value, one at a time, and noting the effect on the results. The parameters studied, values tested and those chosen are presented in Table 3. Simulations with a 1st order scheme were found to show negligible difference indicating that the violation of the Courant condition in a few number of cells for a $0.00005 \mathrm{~s}$ time step does not affect the stability of the solution. As the time step chosen is

Table 3. Numerical parameters tested in the systematic study; medium grid (Table 4). The chosen values are also included.

\begin{tabular}{lll}
\hline Parameter & Tested & Chosen \\
\hline Temporal discretization & 1st/2nd Order & 2nd order \\
Under-Relaxation: Pressure & $0.3,0.4,0.5,0.6$ & 0.4 \\
Under-Relaxation: Velocity & $0.7,0.8,0.9,1.0$ & 0.9 \\
Maximum inner iterations & $8,10,15$ & 10 \\
\hline
\end{tabular}

relatively fine, the influence of the relaxation factor values and number of inner iterations on the pressures was found to be small. The chosen values were, therefore, such that the solution is converged within each time step, i.e. the residuals drop by at least one order of magnitude, and stability in the pressure and velocity fields is attained.

\subsubsection{Effect of mesh size}

A sufficient grid resolution is needed, particularly near the wedge bottom, to accurately capture the highly localized (in space) impact pressures during water entry. Furthermore, the mesh needs to be sufficiently fine such that further refinement won't change the solution (grid independent). A systematic study is performed to assess the influence of the mesh size on the pressures and vertical force. The principal parameters of the three grids tested are presented in Table 4. Refinements were applied to the whole domain and the number of prism layers was adjusted to obtain a smooth transition between the near wall mesh and the core mesh. The grid used in the previous studies (time step and numerical parameters), is referred to here as the 'Medium' grid. The time step size is also adjusted by the same factor (of two) to maintain the same Courant number.

The computed pressure and vertical force time histories on the three grids are shown in Figure 8 and Figure 9 respectively. The coarse grid is not

Table 4. Particulars of the grids tested.

\begin{tabular}{lrlll}
\hline Grid & Cell count & $\begin{array}{l}\Delta \mathrm{x}_{\min }{ }^{*} \\
(\mathrm{~mm})\end{array}$ & $\begin{array}{l}\Delta \mathrm{t}_{\min }{ }^{*} \\
(\mathrm{~mm})\end{array}$ & $\begin{array}{l}\text { Time step size } \\
\Delta \mathrm{t}(\mathrm{s})\end{array}$ \\
\hline Coarse & 8534 & 5 & 5 & 0.0001 \\
Medium & 24174 & 2.5 & 2.5 & 0.00005 \\
Fine & 111365 & 1.25 & 1.25 & 0.000025 \\
\hline
\end{tabular}

*Dimensions of the smallest cell located along the wedge bottom; longitudinal (x-) and tangential (t-) directions.

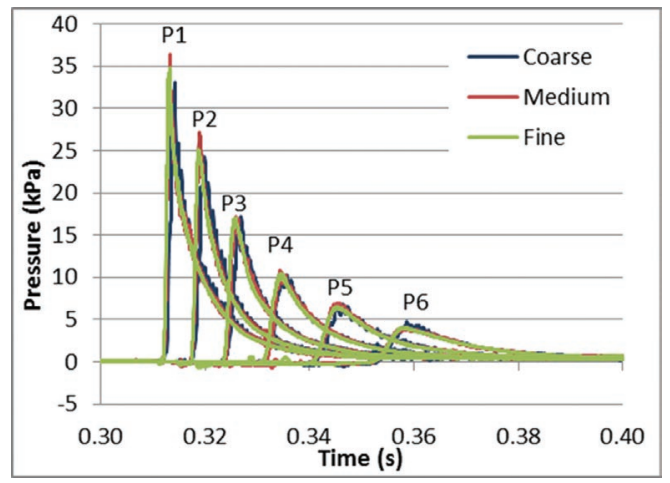

Figure 8. Pressure time histories showing the effects of systematically refining space and time discretisations. 


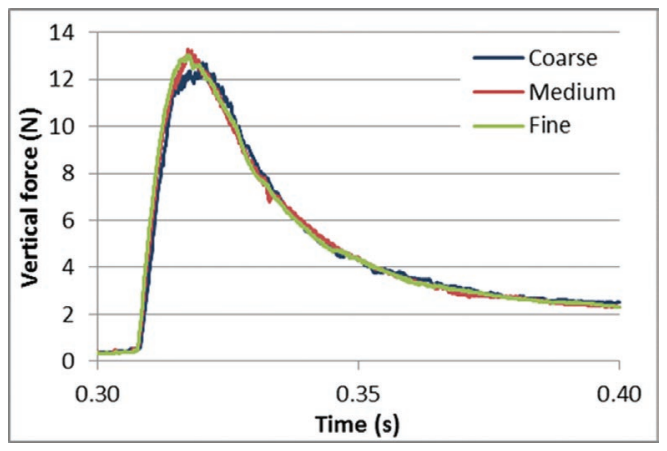

Figure 9. Vertical force time histories showing the effects of systematically refining space and time discretisations.

sufficiently fine to capture the maximum vertical force. The computations on the medium and fine grids agree well, both in terms of shape and magnitude indicating that the influence of the mesh size on the solution is small. The same conclusions can be reached from the pressure plots. The medium and fine grids show good agreement apart from the slight differences in peak values at sensors $\mathrm{P} 1$ and $\mathrm{P} 2$ of $1.5 \mathrm{kPa}$ and $2 \mathrm{kPa}$ respectively, due to reasons discussed earlier. The medium grid is thus chosen for further analysis.

\subsubsection{Validation of numerical model}

The computed pressure and acceleration time histories, using the medium grid with a $0.00005 \mathrm{~s}$ time step size, are shown in Figure 10. The results are low pass filtered with a Butterworth filter built in MATLAB using the same cut-off frequencies as in Lewis et al. (2010). Here, zero time is when the wedge apex reaches the water surface, determined from the high-speed camera images in the experiments and the wedge position plot in the numerical simulations. The initial highest peak is recorded by sensor P1, after which sensors P2 to P6 record progressively lower peaks due to wedge deceleration. Also the shape of the pressure distribution changes from a sharp peak at P1 to a more rounded one at P6. The experimental peak pressures at the time after initial contact with water are also included in Figure 10 for comparison (connected black dots). Compared to the experimental results presented in Figure 3 the computed pressures and acceleration show good agreement in terms of overall shape.

The numerical and experimental peak pressures and time of peak are presented in Table 5. Numerical peak pressures recorded by sensors P3 to P6 agree well with the experiments (difference of less than $0.6 \mathrm{kPa}$ ). However, P1 and P2 are over estimated. There are a number of possible reasons for this discrepancy. For instance, friction between the linear bearings and the vertical posts can have

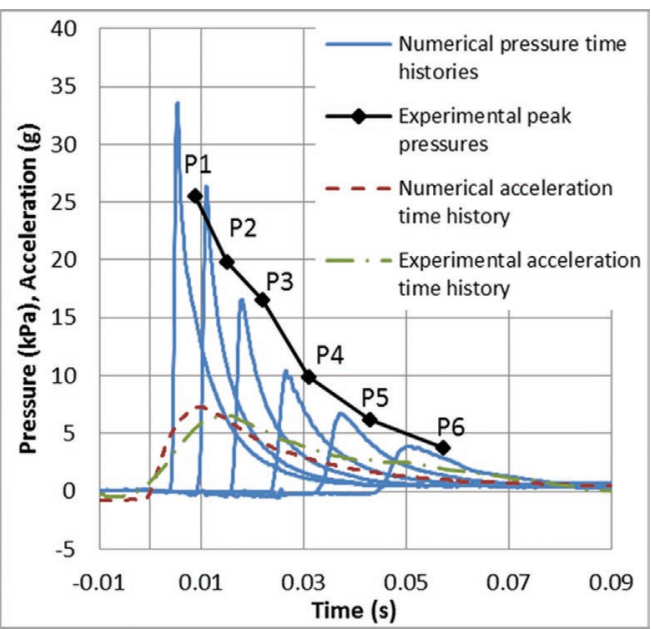

Figure 10. Predicted and experimental pressure and acceleration time histories and peak values; medium grid (Table 4).

Table 5. Numerical and experimental peak pressures and time of peak after impact, medium grid (Table 4).

\begin{tabular}{lllllr}
\hline & \multicolumn{2}{l}{ Experiments } & & \multicolumn{2}{l}{ Simulations } \\
\cline { 2 - 3 } Sensor & $\begin{array}{l}\text { Pressure } \\
(\mathrm{kPa})\end{array}$ & $\begin{array}{l}\text { Time } \\
(\mathrm{ms})\end{array}$ & & $\begin{array}{l}\text { Pressure } \\
(\mathrm{kPa})\end{array}$ & $\begin{array}{r}\text { Time } \\
(\mathrm{ms})\end{array}$ \\
\hline P1 & 25.5 & 8.8 & & 33.6 & 5.3 \\
P2 & 19.8 & 15 & & 26.4 & 10.9 \\
P3 & 16.6 & 21.8 & & 16.6 & 17.8 \\
P4 & 9.96 & 30.9 & & 10.4 & 26.5 \\
P5 & 6.16 & 42.8 & & 6.7 & 37.1 \\
P6 & 3.78 & 57.1 & & 3.9 & 50.9 \\
\hline
\end{tabular}

a significant influence on the wedge velocity, after the initial impact, and, thus, the impact pressures. Unfortunately, the wedge velocity was not measured during the experiments, making it difficult to draw solid conclusions. Nevertheless, the acceleration time histories show some differences (see Figure 10), indicating that the wedge dynamics are not accurately predicted. Secondly, 2D simulations are known to overpredict the impact pressures and forces (Muzaferija et al. 1998). Hydroelastic effects are not believed to have a significant influence, the rigid wedge construction and conditions tested make any influence from elastic effects unlikely.

The time of peak pressure after impact shows less favorable agreement. All numerical sensors record the peak values earlier in time. This difference can be attributed to the uncertainties associated in determining the point of impact in both the experiments and numerical simulations. For instance, Lewis et al. (2010) reports that the 


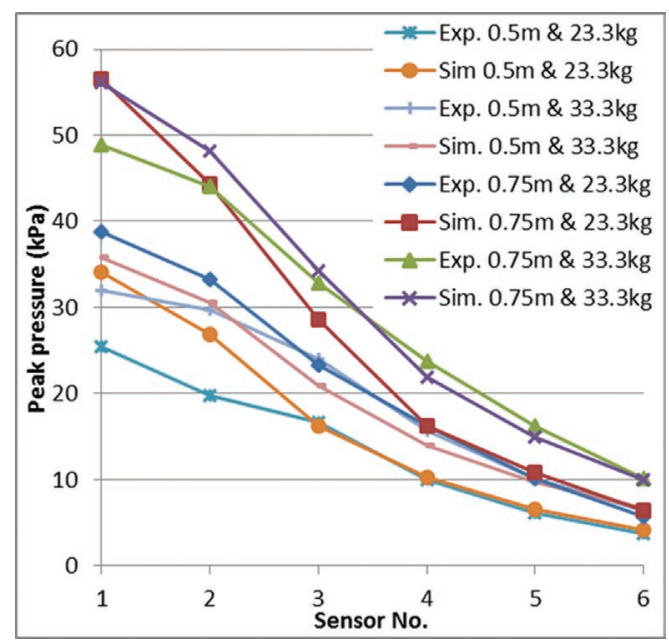

Figure 11. Experimental and numerical peak pressures showing the effects of drop height and wedge mass.

accelerometer begins to be affected by the impact $2.5 \mathrm{~ms}$ after the high speed camera images show the wedge touching the water. During this time the wedge travels approximately $10 \mathrm{~mm}$.

The experimental and numerical peak pressures for the other test configurations, using the medium grid (Table 4) are presented in Figure 11. The drop height has been adjusted for all cases to match the experimental impact velocity. As can be seen, peak pressure increases with increasing impact velocity, as also observed by Wang \& Guedes Soares (2012). Generally speaking, the results for a $33.3 \mathrm{~kg}$ wedge show better agreement with the experiments. This further suggests that friction has a significant influence on the results. In this case, gravitational force is much larger than the frictional forces and the latter will therefore have less influence on the wedge velocity.

\subsubsection{Three-dimensional results}

Three-dimensional simulations were carried out to investigate the influence of these effects on the predictions. Due to symmetry, only one quarter of the experimental tank and wedge is modelled, as shown in Figure 12. The distance between the wedge end and the tank wall (x-direction, width) is $7.5 \mathrm{~mm}$, as in the experiments. The boundary conditions, physics models, numerical parameters and time step are the same as in the 2D simulations. The medium mesh settings were adopted for the three-dimensional grid, with additional refinement in the narrow gap between the wedge end and the tank wall to accurately resolve the flow in this region. The dimensions of the cells are the same in all directions (isotropic mesh refinement). The mesh has

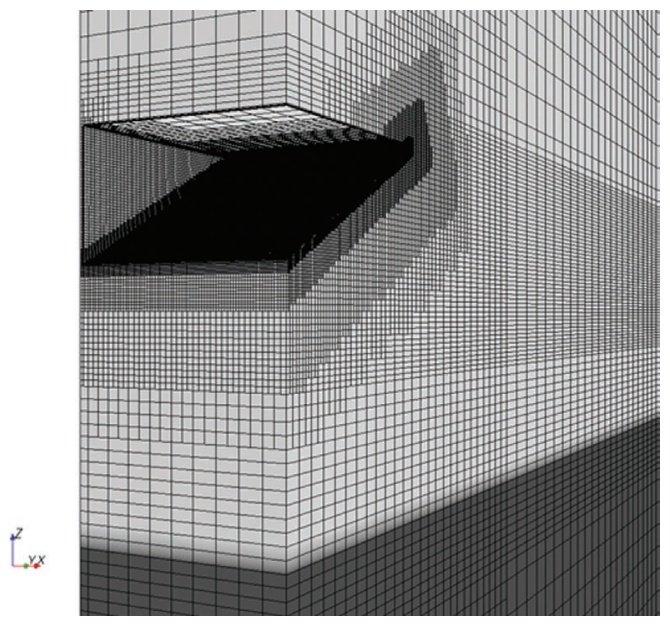

Figure 12. Three-dimensional model of the wedge and tank.

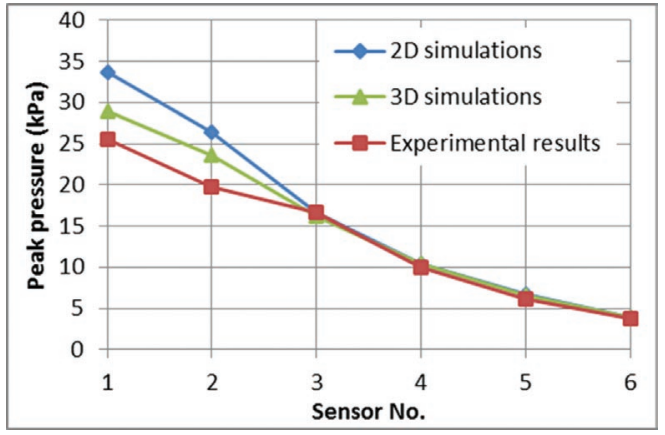

Figure 13. Numerical and experimental peak pressures showing the three-dimensional effects; $0.5 \mathrm{~m}$ drop height, $23.3 \mathrm{~kg}$ mass.

about 520,000 cells. The rigid body velocity time history is the same for the 2D and 3D simulations. The predicted peak pressures for the $3 \mathrm{D}$ case are shown in Figure 13. Two-dimensional results and experimental measurements are included for comparison. Three-dimensional effects have a significant influence particularly during the early stages of water entry. The predicted pressures for sensors $\mathrm{P} 1$ and P2 are lower than those obtained with a two-dimensional grid and tend to compare more favorably with the experimental results (difference of $3.4 \mathrm{kPa}$ and $3.7 \mathrm{kPa}$ respectively).

\section{CONCLUSIONS}

The impact of a rigid wedge with water has been successfully investigated using the commercial CFD software Star CCM+. The influence of mesh 
size, time step and other numerical parameters on the solution has been studied in a systematic manner. This gives more confidence in the numerical model. Sufficient grid and time step resolution is needed to accurately capture the highly localized impact pressures. Results show that a $0.00005 \mathrm{~s}$ time step size and a cell size of $2.5 \mathrm{~mm}$ (along the wedge bottom) is needed to accurately capture the impact pressures. For a sufficiently fine time step, numerical parameters such as relaxation factors and iterations were found to have a secondary effect on the results. The chosen values were such that the solution is converged within each time and stable. The predicted impact pressures were compared with the experimental measurements of Lewis et al. (2010). The numerical model is well capable of predicting the impact event, although the peak pressures at $\mathrm{P} 1$ and $\mathrm{P} 2$ are slightly over estimated. Three-dimensional simulations were found to provide better agreement with the experiments indicating that some three-dimensional effects are present. However, slight discrepancies still exist, which are believed to be due to mechanical friction in the experiments. Nevertheless, it is concluded that the numerical model is sufficiently accurate to allow it to be coupled with a structural solver for further study on hydroelastic impacts.

\section{REFERENCES}

Aarsnes, J., 1996. Drop test with ship sections - effect of roll angle. MARINTEK report.

Brizzolara, S., Couty, N., Hermundstad, O., Ioan, A., Kukkanen, T., Viviani, M. \& Temarel, P., 2008. Comparison of experimental and numerical loads on an impacting bow section. Ships Offshore Struct., 3(4), pp. 305-324.

Lewis, S.G., Hudson, D.A., Turnock, S.R.\& Taunton, D.J., 2010. Impact of a free-falling wedge with water: synchronized visualization, pressure and acceleration measurements. Fluid Dyn. Res., 42(3), pp. 1-30.
Muzaferija, S., Peric, M., Sames, P. \& Schellin, T., 1998. A two-fluid Navier-Stokes solver to simulate water entry. Proc. 22nd Symp. Nav. Hydrodyn., pp. 638-651.

Oger, G., Doring, M., Alessandrini, B. \& Ferrant, P., 2006. Two-dimensional SPH simulations of wedge water entries. J. Comput. Phys., 213(2), pp. 803-822.

Piro, D. \& Maki, K., 2013. Hydroelastic analysis of bodies that enter and exit water. J. Fluids Struct., 37, pp. $134-150$.

Reddy, D.N., Scanlon, T. \& Kuo, C., 2002. Prediction of Slam Loads on Wedge Section using Computational Fluid Dynamics (CFD) Techniques. In 24th Symp. Nav. Hydrodyn. Fukuoka, JAPAN, pp. 706-718.

Sames, P.C., Schellin, T.E., Muzaferija, S. \& Peric, M., 1999. Application of a two-fluid finite volume method to ship slamming. J. Offshore Mech. Arct. Eng., 121(1), pp. 47-52.

Stenius, I., Rosén, A. \& Kuttenkeuler, J., 2006. Explicit FE-modelling of fluid-structure interaction in hullwater impacts. Int. Shipbuild. Prog., 53, pp. 103-121.

Tveitnes, T., Fairlie-Clarke, A.C. \& Varyani, K., 2008. An experimental investigation into the constant velocity water entry of wedge-shaped sections. Ocean Eng., 35(14-15), pp. 1463-1478.

Veen, D. \& Gourlay, T., 2012. A combined strip theory and Smoothed Particle Hydrodynamics approach for estimating slamming loads on a ship in head seas. Ocean Eng., 43, pp. 64-71.

Wagner, H., 1932. Phenomena associated with impacts and sliding on liquid surfaces. Z. Angew. Math. Mech.

Wang, S. \& Guedes Soares, C., 2012. Analysis of the water impact of symmetric wedges with a multi-material Eulerian formulation. Int. J. Marit. Eng., 154(A4), pp. 191-206.

Yettou, E.-M., Desrochers, A. \& Champoux, Y., 2006. Experimental study on the water impact of a symmetrical wedge. Fluid Dyn. Res., 38(1), pp. 47-66.

Zhao, R., Faltinsen, O. \& Aarsnes, J., 1996. Water entry of arbitrary two-dimensional sections with and without flow separation. In Proc. 21st Symp. Nav. Hydrodyn. pp. $408-423$. 